\title{
Removable Prosthesis with Extra Coronal Attachment for the Management of Distal Extension Case: A Case Report
}

\author{
Thapa $\mathrm{D}^{1 *}$, Shrestha $\mathrm{P}^{2}$ \\ ${ }^{1}$ Cosultant Prosthodontist \\ ${ }^{2}$ Dental Surgeon \\ Delight Dental Care, Kalimati, Kathmandu, Nepal
}

\begin{abstract}
Even though dental attachments have a long successful history, many of the dental practioners are unaware about its benefits in daily clinical practice. It highly improves the comfort, aesthetic, function and patient satisfaction, especially in the patients with long span edentulism where implants and fixed partial denture (FPD) are not indicated and cast partial dentures are barely satisfactory. This case report describes the clinical and the laboratory steps for prosthetic rehabilitation of a patient with maxillary distal extension partially edentulism with cast partial denture using Preci Vertex attachments.
\end{abstract}

Key words: Attachment, Distally extended removable partial denture, Preci Vertex

\section{Introduction}

$\mathrm{P}$ recision and semi precision attachments are not a new invention in dentistry. They have been in use in dentistry since early $20^{\text {th }}$ century. ${ }^{1}$ An attachment is a connector consisting of two or more components. One component is connected to a tooth, tooth root or an implant and the other component is connected to prosthesis. ${ }^{2}$

There are two types of attachments:

1. Precision attachments: They are prefabricated, often milled from alloys and are very precise. They are soldered or welded to crown/coping or prosthesis. Generally they are rigid. ${ }^{3}$

2. Semi-precision attachments: They are usually available in plastic forms and are cast, hence less precise. To improve the retention, nylon grips are provided thus are more resilient, easy to repair and less expensive, if casted in base metal alloy.

\section{*Corresponding Author}

Dr. Dipak Thapa, Cosultant Prosthodontist

Delight Dental Care, Kalimati, Kathmandu, Nepal

E-mail:drthapa@icloud.com
Based on location, attachments can also be classified as:

1. Intracoronal attachments: They are placed within the contour of the crown. Hence, they require a huge box preparation and often demand endodontic treatment. Most of them are key and key-way type and are rigid. e.g. Crismani attachments.

2. Extracoronal attachments: They are placed outside the crown contour; thus, more conservative tooth preparation. Mac Collum, Stern Latch, Dalbo, Duo-lock are some of the most commonly used extracoronal precision attachments and ERA, Preci Sazix, Preci Vertex are most commonly used extracoronal semi precision attachments. ${ }^{4,5}$

3. Bar: They span on an edentulous area and connect abutment teeth, roots or implants. The removable bridge, partial denture or over denture fits over the bar with one or more retentive sleeves, riders/clip or retentive plunger. Preci-Bars (Dolder) and Ackermann Bars are examples of precision bars and Hader Bar is Semi Precision Bar. ${ }^{4}$ 
4. Studs: Radicular and intra-radicular stud type attachments are connected to a root preparation; radicular over the root and intra-radicular fits within the root form contour. The Zest Anchor and ZAAG are popular intra-radicular type of attachments whereas, Preci Ball and $\mathrm{O}$ Ring are radicular attachments directly cemented into the prepared root without requiring a cast coping. ${ }^{5,6,7}$

Based on stiffness of the resulting joint, attachments can also be classified as Rigid and Resilient. Rigid types are used as connectors of the fixed partial dentures and sometimes in fully tooth/implant supported removal partial dentures. ${ }^{3}$ In case of tooth-tissue supported prosthesis, resilient types are highly recommended to avoid torque to the abutment tooth. Resiliency of the joint could be in one direction (mostly vertical) or in multiple directions (vertical, true hinge and lateral movements). ${ }^{3}$

Mode of retention of these attachments could be Frictional (between close fitting parallel surfaces), Mechanical (some sorts of physical undercuts), Frictional and Mechanical or Magnetic (magnetic attraction).

Selecting the appropriate attachment for the particular case is often a tedious job. Factors that determine the choice of attachment depends upon types of prosthesis (partial denture, over denture, segmented bridgework), location of the attachment (intracoronal, extracoronal, radicular, intra-radicular), function (rigid or resilient), retention (frictional, mechanical or magnet), space available (occluso-cervical height, buccolingual width, mesio-distal distance) and cost.

Many of the dental practioners in Nepal are still not familiar with the attachments. The major reasons for this could be unavailability of the attachment systems in local market, cost factor and lack of trained lab technicians. If one could overcome these lacunae, then he/ she can add a new dimension to the treatment options, especially for distal extension partially edentulous situations and over denture patients. ${ }^{2}$

\section{A Case report:}

A 67-years-old female was referred for the rehabilitation of maxillary arch. On examination, only few maxillary anterior teeth $(13,11,21$, 22 and 23) were remaining. The maxillary arch was classified as Kennedy's Class I partially edentulous arch.

After oral prophylaxis, endodontic treatment were carried on for teeth 21, 22 and 23. Cast metal post and core were planned for 22 and 23 as clinical crown height were less due to attrition. Porcelain fused to metal (PFM) crown on 11 was removed and all maxillary anterior teeth were prepared for FPD. Maxillary impression was made with Polyvinyl siloxane (PVS) impression material using putty wash technique and mandibular impression was made with reversible hydrocolloid impression material.

Maxillary putty wash impression was poured twice. The first cast was used for fabrication of framework for FPD. Die sectioning was done and working cast with removable dies were made using die lock tray second cast was used as a master cast and was mounted in the articulator using maxillary bite block.

Wax pattern was prepared for PFM Bridge. With the help of dental surveyor and paralleling mandrel, a castable plastic male component of Preci Vertex (Preat Corporation) was attached to the pattern, on the distal aspect of each distal abutment. Using a milling bur, shoulder for lingual bracing arms were prepared on the lingual aspect of the wax patterns for distal abutments. Wax pattern was then invested, casted and finished. 
Metal framework for FPD was tried for accuracy of fit. Framework was veneered with porcelain. Porcelain try-in was done and necessary adjustment and contouring were made, after which the FPD was polished and glazed. Special care must be taken while polishing male components of the Preci Vertex.

Burn out plastic housings was taken and yellow female components were assembled into it. The housings were adapted to proper vertical height and gingival contour and fitted to the male components. Lingual bracing arm were waxed up directly to the housing and further shaping were done to achieve aesthetic and support for the porcelain veneering. Housings were sprued and then removed from the male components. Yellow female components were removed using pointed instrument and then they were invested, casted and finished. The inside of the housings were just sandblasted. After confirming the fit of male and female components, the housings were veneered with porcelain.
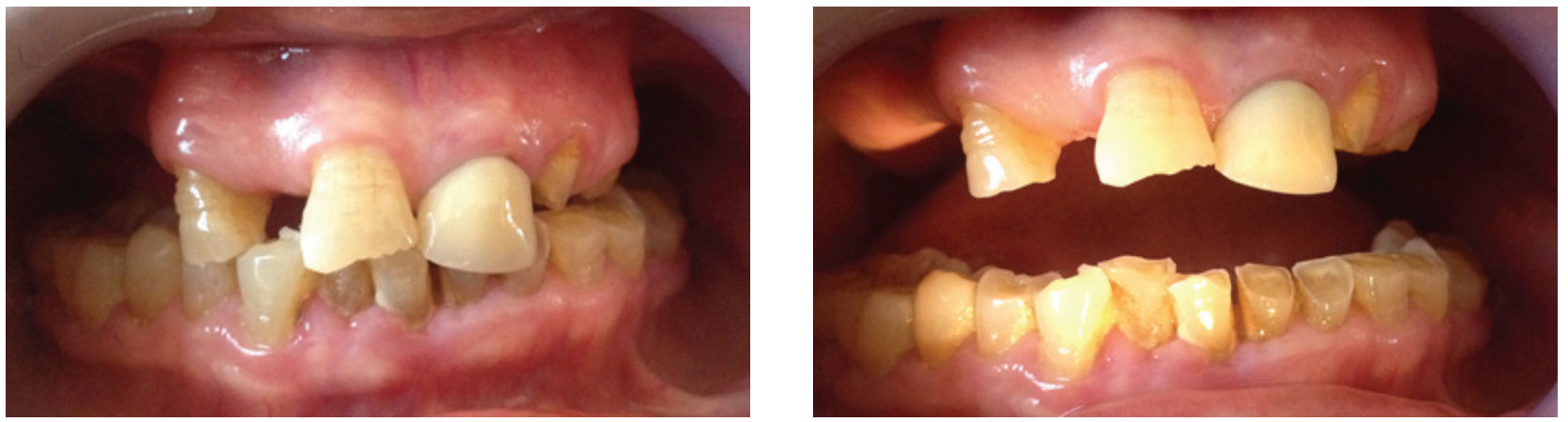

Figure 1 \& 2: Intraoral pre-operative view

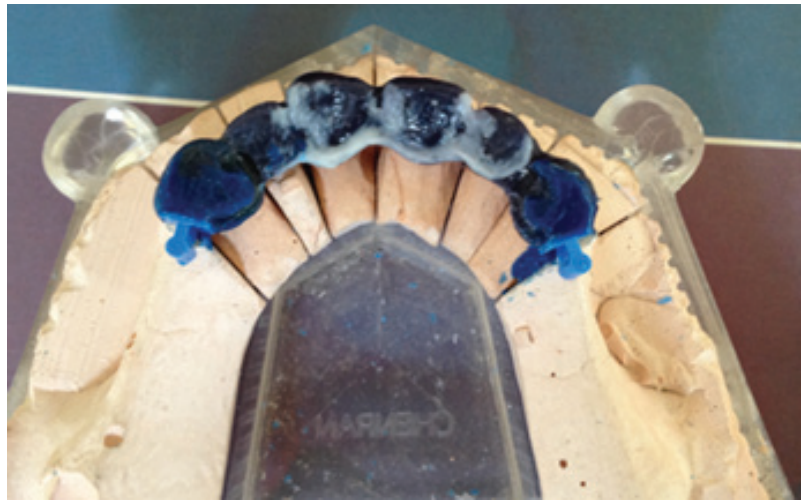

Figure 3: Wax up of maxillary anterior bridge with male component (Preci Vertex)
In the meantime, a framework of maxillary cast partial denture with anterior and posterior palatal strap was also casted. Now we had three components to be assembled: FPD with male components, Female housings with yellow riders in it and Cast partial denture framework.

The housings with yellow females were fitted to the male components. CPD framework was then seated onto the master cast. With the help of flowable composite the extension of the housing was attached to the CPD framework. Acrylic teeth were set up in the articulator and then try in was done. The denture was cured, finished and polished.

FPD was cemented to the abutment teeth with the help of luting GIC. The denture with housing and new yellow female component was delivered. Post insertion instructions were given and patient was kept on regular follow up appointments. 


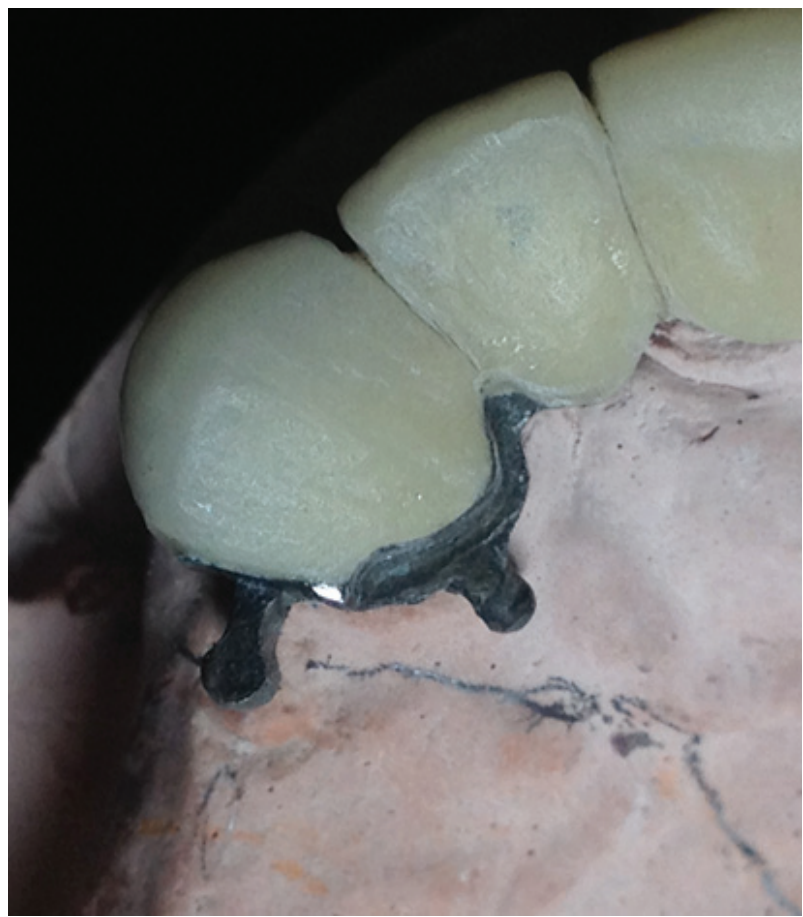

Figure 5: Close up view of male component and lingual shoulder

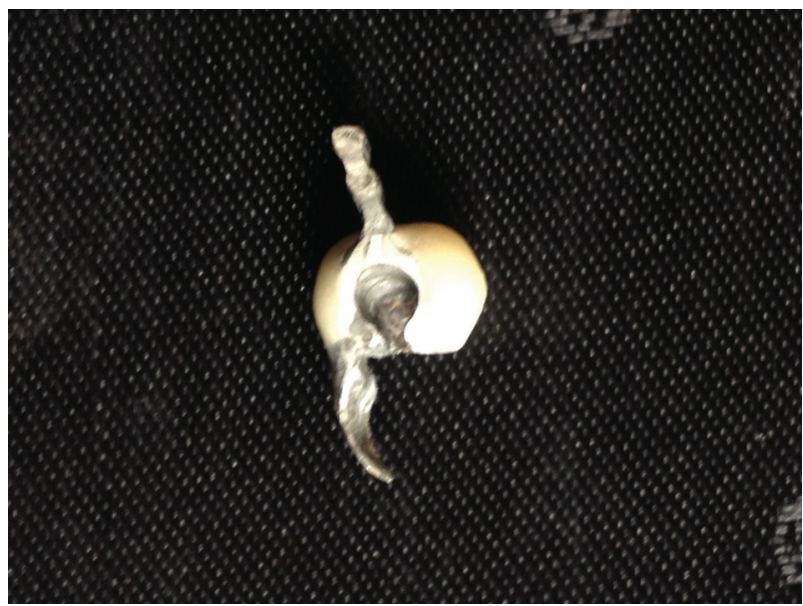

Figure 6: Female housing with lingual bracing arm

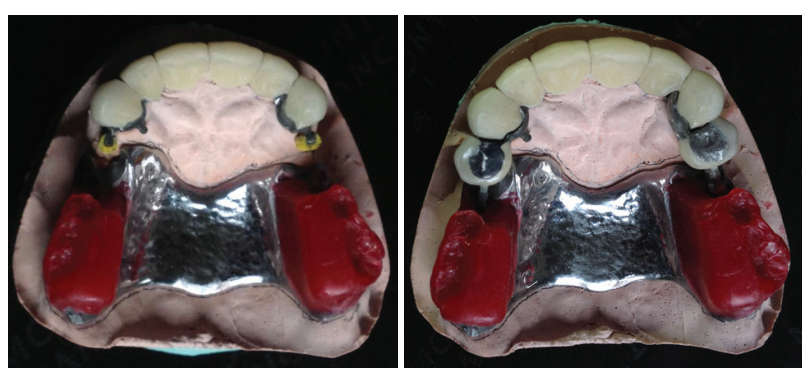

Figure 7 \& 8: PFM bridge, yellow retentive grip over male components, female housing ready to be connected to the denture framework

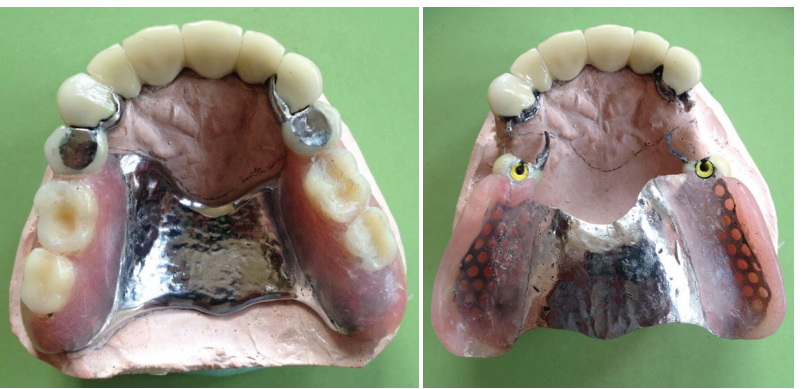

Figure $9 \&$ 10: After processing of the denture

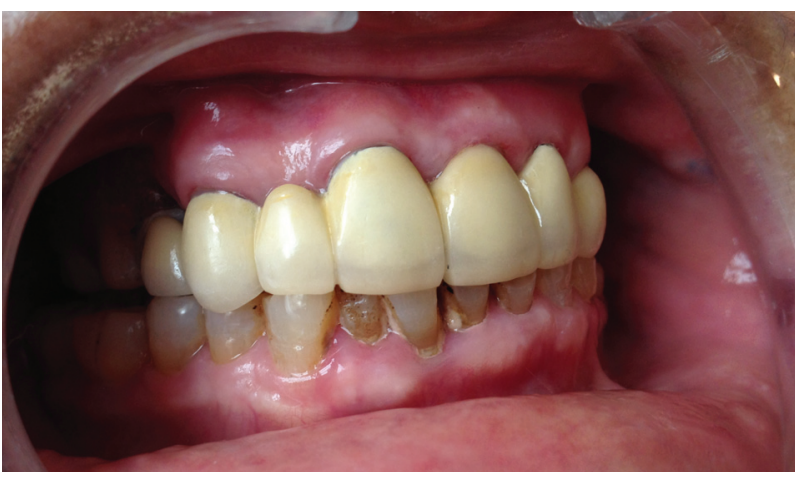

Figure 11: Post operative view with all components in the mouth

\section{Discussion}

Rehabilitation of the patients with distal extension partially edentulous arches is one of the most challenging situations that a dentist encounters in clinical practice. There is no doubt that the implant supported FPD would be the optimum treatment option but due to surgical and financial limitation many patients are reluctant for implant supported partial dental prosthesis. Dental precision and semiprecision attachments are the viable alternatives in such cases. $^{1}$

Cast partial denture has been the treatment of choice for decades for distal extension cases but when the most distal abutment teeth are in anterior aesthetic zone, clasp assembly potentially interferes with the aesthetic. In such cases, attachments are superior alternatives as they are esthetically superior. ${ }^{1}$

Attachments for the distal extension cases have to be resilient type with both vertical and lateral movements. Under occlusal loading torque 
force should not be transferred to the abutment teeth. It is also recommended to splint the distal abutments with mesial tooth for better support. ${ }^{3}$ The use of lingual shoulder and bracing arms are highly recommended in most of the extracoronal attachments, especially in distal extension partially edentulous cases. ${ }^{5}$ They not only guide in placement and removable of the prosthesis but also improve the stability of the dentures.

Though there is an alternate technique but the use of green burn out plastic housing makes the job easy and precise. Preci Vertex even supplies $\mathrm{Co} / \mathrm{Cr}$ metal housing. ${ }^{5}$ The yellow female may lose its retentiveness due to wear from usages and they needs to be replaced periodically. ${ }^{8}$ It is often required to pick up the FPD with pick-up impression because the fit of FPD in the patient's mouth may differ from the cast occasionally. It is easier and better to join the female housing with the CPD framework inside the patient's mouth rather than on the master cast. In worst scenario, the denture may need to be relined by some chair side relining materials.

\section{Conclusion}

Clinical and laboratory procedures for dental attachments may seem to be lengthy, costly and complicated but with the knowledge of basic prosthodontics, good clinical skills and judgment one can implement the use of attachments in daily clinical practice. This will highly improve the comfort, aesthetic, function and satisfaction of the patients with long span edentulism where implants and FPDs are not indicated.

\section{References}

1. Shakeel SK. Removable prosthesis using extracoronal precision attachment. Gulf Med J, 2013;2:126-129.

2. Glossary of Prosthodontic Terms, Ninth Edition, J Prosthetic Dent 2016;117:13-14

3. Nigam A, Singh A et al. Precision Attachments: an overview. J Dent Facial Sci 2013; 2: 41-44

4. Bambara GE. Precision and Semi-Precision Attachments. In Freedman GA. Contemporary Esthetic Dentistry $1^{\text {st }}$ ed. Elsevier, chapter $25 \mathrm{p}$ 575.

5. “About Us: Ceka Preci line," (online). Available: www.ceka-preciline.com.

6. Sterngold Attachments. (online). Available: www.sterngold.com

7. Becerra $G$ et al. A classification of precision attachments. J Prosthet Dent 1987: 56: 322327.

8. Prashad KD et al. A Simplified Approach to Semi Precision Attachment. NUJHS 2016,6:5157. 